\title{
BTF Modelling using BRDF Texels
}

\author{
J. Filip \& M. Haindl \\ \{filipj,haindl\}@utia.cas.cz
}

Dept. of Pattern Recognition, Institute of Information Theory and Automation, Academy of Sciences of the Czech Republic, Prague, Czech Republic

\begin{abstract}
The highest fidelity representations of realistic real-world materials currently used comprise Bidirectional Texture Functions (BTF). The BTF is a six dimensional function depending on view and illumination directions as well as on planar texture coordinates. The huge size of such measurements, typically in the form of thousands of images covering all possible combinations of illumination and viewing angles, has prohibited their practical exploitation and obviously some compression and modelling method of these enormous BTF data spaces is inevitable. The proposed approach combines BTF spatial clustering with cluster index modelling by means of an efficient Markov random field model. This method allows to generate seamless cluster index of arbitrary size to cover large virtual $3 \mathrm{D}$ objects surfaces. The method represents original BTF data using a set of local spatially dependent Bidirectional Reflectance Distribution Function (BRDF) values which are combined according to synthesised cluster index and illumination / viewing directions. BTF data compression using this method is about $1: 100$ and their synthesis is very fast.
\end{abstract}

\section{Introduction}

Recent progress in graphics hardware computational power finally enables fast and visually realistic rendering of virtual reality models that until recently was impossible. Such realistic models require, among others, natural looking textures covering virtual objects of rendered scene. Applications of these advanced texture models in virtual reality systems now allow photo-realistic material appearance approximation for such complex tasks as visual safety simulations or interior design in automotive/airspace industry or architecture.

For the aim of such advanced applications a smooth textures lit by reflectance models alternatively combined with bump-mapping are not able to offer correct and realistic reproduction of material appearance. This is caused due to inherited complexity of many materials whose rough structure produces such visual effects as self-shadowing, masking, inter-reflection or subsurface scattering. The one way to capture these material's attributes is using much more complex representation of a rough or 3D texture called Bidirectional Texture Function (BTF). BTF is a six dimensional function depending on view and illumination directions as well as on planar texture coordinates as illustrated in Fig.1. This function is typically acquired in the form of several thousands images covering varying light 
and camera directions. However, a huge size of measured BTF data prevents their usage in any useful application so introduction of some fast compression and modelling method for BTF data is inevitable.

The majority of results in the BTF area deal mainly with compression. They are based either on eigen-analysis of BTF data space [1-4] or on applications of pixel-wise reflectance models [5-8]. Although these methods can provide reasonable compression ratios $\left(\frac{1}{20}-\frac{1}{200}\right)$ and visual quality, their main drawback is that they do not allow arbitrary size BTF synthesis, i.e. the texture enlargement.

To solve this problem additional BTF enlargement methods are necessary. Unfortunately there are not many BTF enlargement approaches available. A majority of the available methods are based either on simple texture repetition with edge blending or on more or less sophisticated image tiling methods [9-12] and they can be adapted also for BTF synthesis, e.g., [13].

Finally a group of probabilistic BTF models was recently proposed [14], [15]. These methods allow unlimited texture enlargement, BTF texture restoration, huge BTF space compression and even modelling of previously unseen BTF data. They are based on rough BTF segmentation in a space of illumination and viewing directions. The individual clusters representatives are BTF images closest to cluster centers, which are combined with estimated range-map in bump-mapping filter for required illumination and viewing angles. Although these methods reach huge impressive compression ratios they sometimes compromise visual quality for certain materials. In this paper we present a novel BTF model enabling

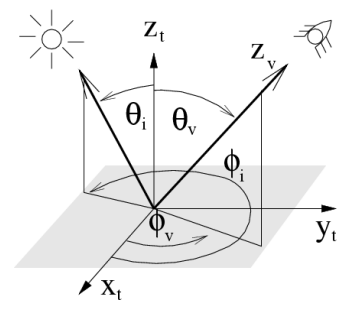

Fig. 1. Relationship between illumination and viewing angles within texture coordinate system.

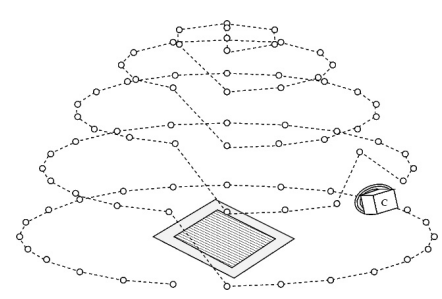

Fig. 2. Illumination directions $(i=1 \ldots 81)$ in used BTF data. Viewing directions $(v=$ $1 \ldots 81)$ are the same.

seamless enlargement of BTF data. The overall scheme of the proposed model is illustrated in Fig.3. The method starts with normal-map estimation of the underlying material surface using photometric stereo. The estimated normalmap $\mathbf{N}$ is enlarged to the required size using probabilistic MRF model. In the following step the original BTF data are clustered in the spatial planar space. The results are cluster representatives $\mathbf{C}$ and cluster index $\mathbf{I}$, which is used for new cluster index $\mathbf{I}_{S}$ generation up to the size of synthesised normal-map $\mathbf{N}_{S}$. This enlargement exploits matching between estimated $\mathbf{N}$ and synthesised $\mathbf{N}_{S}$ normal-maps and BRDFs at neighbouring spatial locations.

This paper is organised as follows. The spatial BTF data segmentation is described in Section 2, the surface geometry estimation (normal-map) is described 


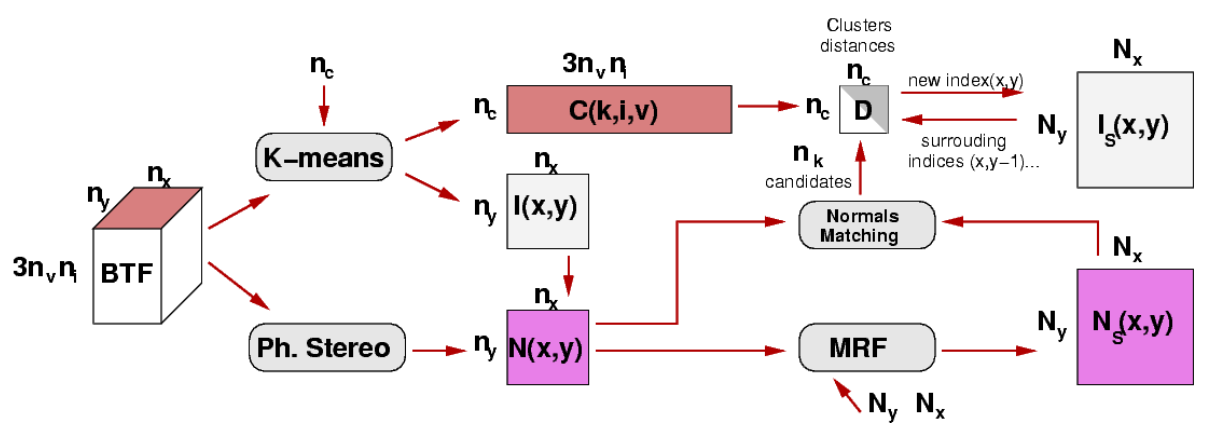

Fig. 3. The overall scheme of the proposed BTF enlargement method.

in Section 3. The surface geometry synthesis using MRF model is subject of Section 4, while the final BTF data enlargement step is described in Section 5. Following sections show results of the proposed model, discuss its properties and conclude the paper.

\section{BTF Space Segmentation}

BTF data employed in this study were measured at the Bonn University [16]. We used BTFs of two different types of lacquered wood. Each dataset comprises 81 viewing positions $n_{v}$ and 81 illumination positions $n_{i}$ (see Fig.2) resulting into 6551 images with resolution (rectified measurements) $800 \times 800$. To decrease computational demands of the following BTF clustering step an image tiling approach was applied. The method [12] finds sub-optimal paths in original data to cut required set of contactable BTF tiles. In our experiments only one BTF tile per material was used.

The input to our algorithm is such a seamless BTF tile in the form of $n_{i} n_{v}$ illumination/view dependent images of size $n_{x} \times n_{y}$. A vector of BTF values for a fixed planar position will be called local BRDF and denoted as BRDF in scope of this paper. In the first preprocessing step all BTF images were converted to CIE Lab perceptually uniform colour space and only data from luminance channel $L$ was used in data vector. The following K-means clustering was performed in the $n_{x} \times n_{y}$ planar space corresponding to individual pixels of BTF. Each pixel represents BRDF of surface geometry at a planar location $(x, y)$. The clustering distance function is:

$$
d(x, y, i, v, k)=\sum_{v=1}^{n_{v}} \sum_{i=1}^{n_{i}}|\mathbf{B}(i, v, x, y)-\mathbf{C}(k, i, v)| \cos \theta_{v}
$$

where $\mathbf{B}(i, v, x, y)$ is the corresponding BTF value, $\mathbf{C}(k, i, v)$ are cluster centers and $i=1 \ldots n_{i}$ and $v=1 \ldots n_{v}$ are illumination and viewing directions of the original BTF data (see Fig.2), respectively. The view elevation angle cosine accommodates the shortening of surface emitting area. The clustering results in the index array $\mathbf{I}(x, y) \in 1 \ldots n_{c}$ and the set of $n_{c}$ cluster representatives 
$\mathbf{C}(k, i, v)$ of the size $n_{c} \times 3 n_{i} n_{v}$ corresponding to the closest colour BRDFs to cluster centers. Note that the individual colour BRDFs representing cluster centers $\mathbf{C}$ correspond to representative set of material locations bearing the most distinct appearance over the BTF tile. Results of the proposed BTF clustering $\left(n_{c}=256\right)$ mapped on 3D object in comparison with original BTF data mapping are shown in the first two rows of Fig.6.

\section{Surface Geometry From BTF}

In order to find smooth spatial representation of the cluster index $\mathbf{I}$ for a further enlargement by means of MRF model we used normal-map describing a geometry of the original material surface. For this purpose the standard photometric stereo technique [17] was applied. This approach is advantageous since the BTF data comprises number of images with fixed viewpoint and variety of defined illumination source directions. As we have much more than three different light positions we used overdetermined photometric stereo. All directions to light sources are ordered in rows of matrix $\mathbf{L}$ and corresponding pixel intensity for different illumination directions are ordered to the vector $\mathbf{E}(x, y)$. Then surface normal-map $\mathbf{N}$ of BTF tile at each pixel was computed by means of the least-squares fitting

$$
\mathbf{N}(x, y)=\frac{\left(\mathbf{L}^{T} \mathbf{L}\right)^{-1} \mathbf{L}^{T} \mathbf{E}(x, y)}{\left\|\left(\mathbf{L}^{T} \mathbf{L}\right)^{-1} \mathbf{L}^{T} \mathbf{E}(x, y)\right\|} .
$$

Alternative approach using range-scanner is costly and does not allow satisfactory measurement of textile materials due to laser beam scattering in material structure.

\section{Probabilistic Normal-Map Modelling}

The smooth texture model based on MRF 3D causal auto-regressive (CAR) model $[18,19]$ was applied to normal-map modelling. The overall scheme of the 3D CAR MRF model is depicted in Fig.4. As an input of the model was image of size $N \times M=512 \times 512$ generated by repetition of the seamless normal-map tile estimated in the previous step.

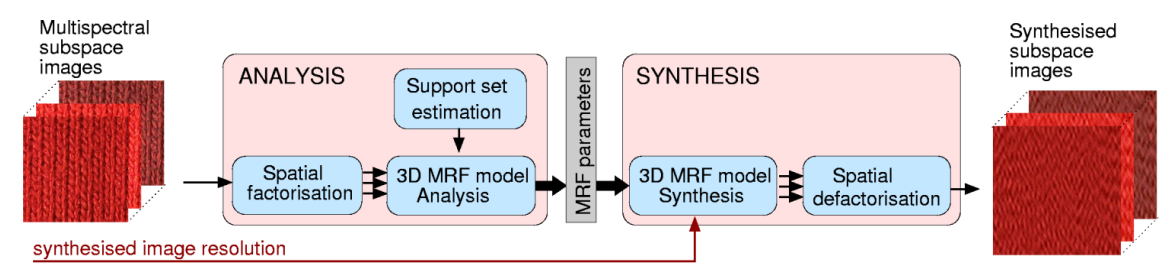

Fig. 4. The overall 3D CAR smooth model scheme. 


\subsection{Spatial Factorisation}

Input tiled normal-map $\bar{Y}_{\bullet}$ (the notation $\bullet$ has the meaning of all possible values of the corresponding index) is decomposed into a multi-resolution grid and each resolution data are independently modelled by their dedicated CAR models. Each one generates a single spatial frequency band of the normal-map. An analysed normal-map is decomposed into multiple resolutions factors using Laplacian pyramid and the intermediary Gaussian pyramid $\ddot{Y}_{\bullet}^{(k)}$ which is a sequence of images in which each one is a low-pass down-sampled version of its predecessor. The Gaussian pyramid for a reduction factor $n$ is

$$
\ddot{Y}_{r}^{(k)}=\downarrow_{r}^{n}\left(\ddot{Y}_{\bullet, i}^{(k-1)} \otimes w\right) \quad k=1,2, \ldots
$$

where $\ddot{Y}_{\bullet}^{(0)}=\bar{Y}_{\bullet}, \downarrow^{n}$ denotes down-sampling with reduction factor $n$ and $\otimes$ is the convolution operation. The convolution mask based on weighting function (FIR generating kernel) $w$ is assumed to execute separability, normalisation, symmetry and equal contribution constrains. The FIR equation is then $\ddot{Y}_{r}^{(k)}=\sum_{i, j=-l}^{l} \hat{w}_{i} \hat{w}_{j} \ddot{Y}_{2 r+(i, j)}^{(k-1)}$. The Laplacian pyramid $\dot{Y}_{r}^{(k)}$ contains band-pass components and provides a good approximation to the Laplacian of the Gaussian kernel. It can be constructed by differencing single Gaussian pyramid layers:

$$
\dot{Y}_{r}^{(k)}=\ddot{Y}_{r}^{(k)}-\uparrow_{r}^{n}\left(\ddot{Y}_{\bullet}^{(k+1)}\right) \quad k=0,1, \ldots,
$$

where $\uparrow^{n}$ is the up-sampling with an expanding factor $n$.

\subsection{D Causal Auto-Regressive Model}

Multi-spectral normal-map was in the previous step decomposed into a multiresolution grid and each resolution data is modelled independently by independent Gaussian noise driven 3D CAR MRF model that enable simultaneous modelling of all resolution factors.

Let the normal map $Y$ is indexed on a finite rectangular three-dimensional $N \times M \times 3$ underlying lattice $I$, where $N \times M$ is the image size. Let us denote a simplified multi-index $r$ to having two components $r=\left\{r_{1}, r_{2}, r_{3}\right\}$. The first component is a row index, the second one is a column index and the third is a normal vector index, respectively. $I_{r}$ specifies shape of the contextual neighbourhood $(\mathrm{CN})$ around the actual index $r=\left\{r_{1}, r_{2}, r_{3}\right\}$. Causality is fulfilled when all data obtained from $\mathrm{CN}$ are known (not missing pixels).

From this causal CN the data are arranged in a vector $X_{r}=\left[Y_{r-s}^{T}: \forall\{s\} \in I_{r}^{c}\right]^{T}$.

The (CAR) random field is a family of random variables with a joint probability density on the set of all possible realisations $Y$ of the $M \times N \times 3$ lattice $I$, subject to the following condition:

$$
\begin{aligned}
p\left(Y \mid \Theta, \Sigma^{-1}\right) & =(2 \pi)^{-\frac{3(M N-1)}{2}}\left|\Sigma^{-1}\right|^{\frac{(M N-1)}{2}} \\
& \exp \left\{-\frac{1}{2} \operatorname{tr}\left\{\Sigma^{-1}\left(\begin{array}{c}
-I \\
\Theta^{T}
\end{array}\right)^{T} \tilde{V}_{M N-1}\left(\begin{array}{c}
-I \\
\Theta^{T}
\end{array}\right)\right\}\right\},
\end{aligned}
$$


where $I$ is identity matrix, $\Theta$ is parameter matrix, $\Sigma$ is covariance matrix of Gaussian white noise and

$$
\tilde{V}_{r-1}=\left(\begin{array}{ll}
\tilde{V}_{Y Y(r-1)} & \tilde{V}_{X Y(r-1)}^{T} \\
\tilde{V}_{X Y(r-1)} & \tilde{V}_{X X(r-1)}
\end{array}\right)
$$

The used notion is $\tilde{V}_{A B(r-1)}=\sum_{k=1}^{r-1} A_{k} B_{k}^{T}$.

Simplified notation $r, r-1, \ldots$ denotes the multi-channel process position in $I$, i.e., $r=\left\{r_{1}, r_{2}, r_{3}\right\}, r-1$ is the location immediately preceding $\left\{r_{1}, r_{2}, r_{3}\right\}$, etc. A direction of movement on the underlying image sub-lattice is common rows scanning. The data from model history obtained during adaptation are denoted as $Y^{(r-1)}$.

The 3D CAR model can be expressed as a stationary causal uncorrelated noise driven $3 \mathrm{D}$ autoregressive process:

$$
Y_{r}=\Theta X_{r}+e_{r}
$$

where $\Theta=\left[A_{1}, \ldots, A_{\eta}\right]$ is the $3 \times 3 \eta$ parameter matrix and $\eta=\operatorname{card}\left(I_{r}^{c}\right)$, $I_{r}^{c}$ is a causal CN, $e_{r}$ is a Gaussian white noise vector with zero mean and a constant but unknown covariance matrix $\Sigma$.

\subsection{Parameter Estimation}

There are two matrices, the parameterer matrix $\hat{\Theta}_{r}$ and the noise covariance matrix $\hat{\Sigma}_{r}$, to estimate / update in each step, i.e., $\mathrm{CN}$ shift on image lattice. Owing to the model causality and the normal-Wishart parameter prior single CAR model parameters (8),(9) can be estimated analytically [19]. The parameter matrix estimate is

$$
\hat{\Theta}_{r-1}^{T}=V_{X X(r-1)}^{-1} V_{X Y(r-1)},
$$

while the covariance matrix estimate is

$$
\hat{\Sigma}_{r-1}=\frac{\lambda_{(r-1)}}{\beta(r)}
$$

where $\lambda_{(r)}=V_{Y Y(r)}-V_{X Y(r)}^{T} V_{X X(r)}^{-1} V_{X Y(r)}, V_{A B(r-1)}=\tilde{V}_{A B(r-1)}+V_{A B(0)}$ and matrices $V_{A B(0)}$ are the corresponding matrices from the normal-Wishart parameter prior. The estimates (8),(9) can be also evaluated recursively if necessary. Where the $\beta(r)=\beta(0)+r-1$ represents number of model movements on image plane $(\beta(0)>1)$.

\subsection{Normal-Map Synthesis}

The CAR model synthesis is very simple and the Markov random field can be directly generated from the model equation (7) with respect to $\mathrm{CN}$ data vector $X_{r}$ and the estimated parameter matrix $\hat{\Theta}_{r}$ using a multivariate Gaussian whitenoise generator. The fine-resolution normal-map is obtained from the pyramid collapse procedure, which is inverse process to the spatial factorisation (3),(4) described in Section 4.1. The comparison of synthesised normal-maps $\mathbf{N}_{S}$ with their originals $\mathbf{N}$ is illustrated in the first row of Fig.5. 


\section{$5 \quad$ New Cluster Index Synthesis}

New cluster index $\mathbf{I}_{S}$ is obtained by row-wise scanning of synthesised normal-map $\mathbf{N}_{S}$. For each normal in the $\mathbf{N}_{S}$ the $n_{k}$ closest normals from normal-map $\mathbf{N}$ of original BTF tile is determined with respect to the Euclidean metric between two unite vectors. However, this approach alone is unsatisfactory because it allows ambiguous normals assignment owing to the material surface. For instance, a normal vector pointing straight upwards can represent either a peak or a valley on the surface. Thus, if a new index is created only based on normal matching the resulted enlarged BTF images are very noisy, while the synthesised structure of normal-map is considerably suppressed. To improve a spatial continuity of generated new cluster index we used information of surface height, occlusion and masking of surface points which is hidden in colour BRDFs of individual stored clusters C. Individual cluster indices corresponding to candidate normal $k$ from $\mathbf{N}$ are obtained from the same $(x, y)$ location from $\mathbf{I}$ as is the spatial location of the normal $k$. From obtained $n_{k}$ normal candidates from the original index $\mathbf{I}$ the optimal one $k^{*}$ is chosen that minimise distance $D$ between the candidate's BRDF and the BRDFs of its surrounding pixels at the locations $(x, y-1)$ and $(x-1, y)$ from the causal neighbourhood in $\mathbf{I}_{S}(10)$

$$
k^{*}=\arg \min _{k=1 \ldots n_{c}}\left(\mathbf{D}\left(\mathbf{I}\left(x_{k}, y_{k}\right), \mathbf{I}_{S}(x, y-1)\right)+\mathbf{D}\left(\mathbf{I}\left(x_{k}, y_{k}\right), \mathbf{I}_{S}(x-1, y)\right)\right) .
$$

To speed up this process a mutual distances between each couple of $n_{c}$ clusters is precomputed (11) and stored in a form of matrix $\mathbf{D}$ of size $n_{c} \times n_{c}$

$$
\mathbf{D}(a, b)=\sum_{v=1}^{n_{v}} \sum_{i=1}^{n_{i}}|\mathbf{C}(a, i, v)-\mathbf{C}(b, i, v)| \cos \theta_{v} .
$$

The $\left(x_{k^{*}}, y_{k^{*}}\right)$ position in new index $\mathbf{I}_{S}$ is obtained by means of $\mathbf{I}_{S}(x, y)=$ $\mathbf{I}\left(x_{k^{*}}, y_{k^{*}}\right)$ using the clusters indices from original index I. Proposed matching scheme incorporates such effects as masking and occlusions and together with normals matching enable reliable and perceptually correct spatial ordering of individual clusters in new enlarged index $\mathbf{I}_{S}$. Additionally, this ordering enforces continuity constraint by placement of the similar BRDFs into neighbouring positions in generated cluster index $\mathbf{I}_{S}$.

For BTF rendering from the proposed model the cluster representatives $\mathbf{C}$ and synthesised cluster index $\mathbf{I}_{S}$ have to be stored enabling compression ratio approximately $\frac{1}{100}$ (for $n_{c}=256$ ). The required BTF value is obtained as

$$
B T F(x, y, i, v)=\mathbf{C}\left(\mathbf{I}_{S}(x, y), i, v\right) .
$$

An example of BTF images synthesised from the model for both tested materials compared with original BTF tiles is shown in the second row of Fig.5.

\section{Results}

The proposed method was applied to BTF enlargement of two different types of smooth lacquered wood. The original BTF tile of wood01 have size $122 \times$ 


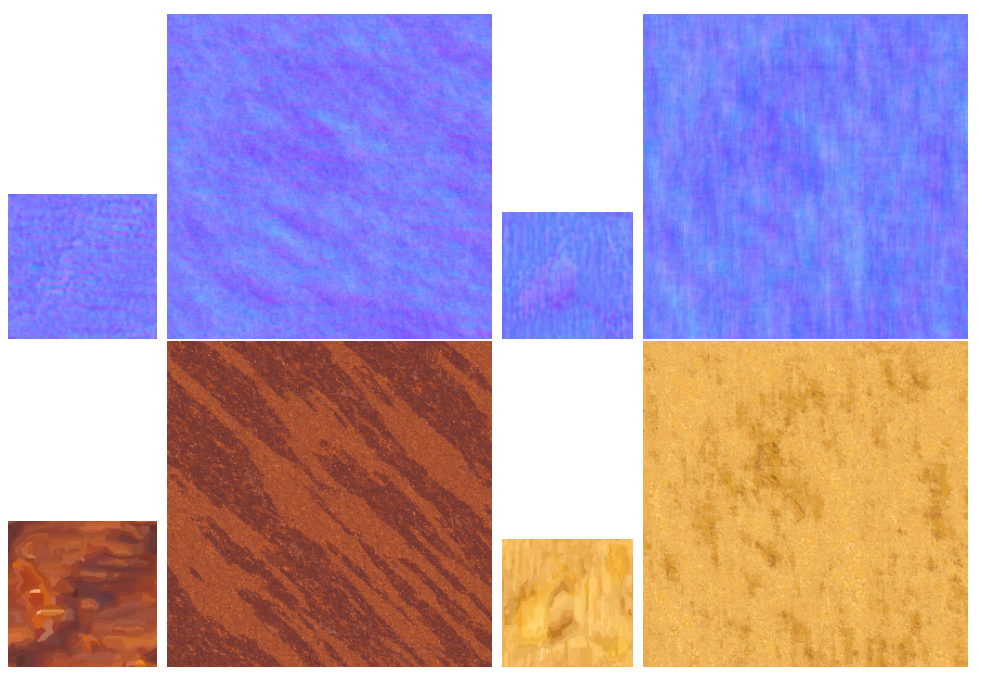

Fig. 5. The first row: Estimated normal tiles (small) and their synthesised counterparts (large) for wood01 (left) and wood01 (right). The second row: clustered BTF tiles for $\theta_{i}=15^{\circ}, \phi_{i}=180^{\circ}, \theta_{v}=0^{\circ}, \phi_{v}=0^{\circ}$ (small) and corresponding BTF images synthesised using enlarged cluster index $\mathbf{I}_{S}$ (large).

125 and for wood02 it is $137 \times 142$. The size of synthesised normal-maps and subsequently index arrays was for both the materials $300 \times 300$. Example of single planar BTF image enlarged by the proposed method is shown in the second row of Fig.5. Comparison of the enlarged BTF data mapped on 3D object with original BTF tile mapping is shown in Fig.6. The interpolation for arbitrary (non-measured) illumination and viewing angles was performed by means of barycentric coordinates [20]. The time demands of the analytical part of the proposed method are not too important since the BTF segmentation, normalmap estimation and synthesis and finally estimated and synthesised normals matching are offline tasks. The most time-consuming part of the method is BTF tile clustering that takes approximately one hour when using $n_{c}=256$ clusters for BTF tile of wood02, while the remaining analytical steps are much faster, depending on the size of original and required normal-map. For BTF tile of wood02 and required new cluster index $\mathbf{I}_{S}$ size $512 \times 512$ it takes several seconds only. All experiments were performed on PC Athlon 1.9GHz, 2GB RAM. A compression ratio of the proposed method for 256 clusters is approximately $\frac{1}{100}$.

\section{Summary and Conclusions}

This paper proposes new technique for seamless BTF data enlargement. The method strictly separates analytical offline part from the fast possibly real-time synthesis part of the modelling process. The BTF clustering allows to trade-off compression ration and visual quality. The method shows the best performance for spatially random i.e. non-regular types of BTFs such as the tested lacquered 


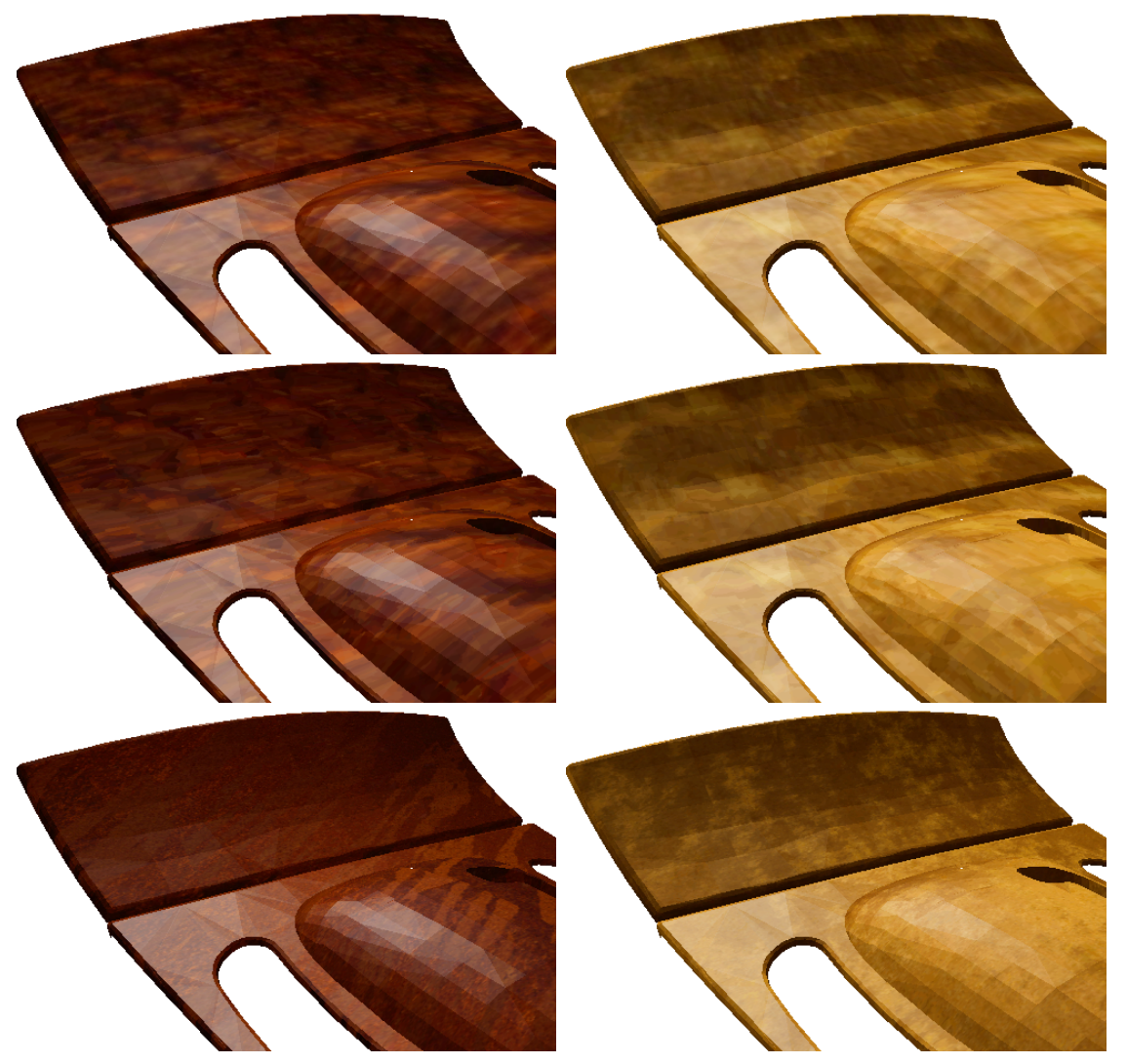

Fig. 6. Results of the proposed BTF data enlargement method mapped on 3D object (third row) in comparison with one original BTF tile mapping (first row) and its segmentation into $n_{c}=256$ clusters (second row) for two kinds of lacquered wood.

wood or leather, etc. The method enables fast seamless BTF data enlargement to arbitrary size with minimal additional storage requirements since the number of clusters is fixed.

\section{Acknowledgements}

This research was supported by the EC project no. FP6-507752 MUSCLE and partially by the grants no. A2075302, 1ET400750407, of the Grant Agency of the Academy of Sciences CR, and MSMT project no. 1M0572.

\section{References}

1. Koudelka, M., Magda, S., Belhumeur, P., Kriegman, D.: Acquisition, compression, and synthesis of bidirectional texture functions. In: Proceedings of the 3rd International Workshop on texture analysis and synthesis (Texture 2003). (2003) $47-52$ 
2. Vasilescu, M., Terzopoulos, D.: TensorTextures: Multilinear image-based rendering. ACM SIGGRAPH 2004, ACM Press 23(3) (2004) 336-342

3. Sattler, M., Sarlette, R., Klein, R.: Efficient and realistic visualization of cloth. In: Eurographics Symposium on Rendering 2003. (2003)

4. Müller, G., Meseth, J., Klein, R.: Compression and real-time rendering of measured BTFs using local PCA. In: Vision, Modeling and Visualisation 2003. (2003)

5. McAllister, D.K., Lastra, A., Heidrich, W.: Efficient rendering of spatial bidirectional reflectance distribution functions. Graphics Hardware (2002) 77-88

6. Malzbender, T., Gelb, D., Wolters, H.: Polynomial texture maps. In: ACM SIGGRAPH 2001, ACM Press, Eurographics Association, Switzerland (2001) 519-528

7. Meseth, J., Müller, G., Klein, R.: Preserving realism in real-time rendering of bidirectional texture functions. In: OpenSG Symposium 2003, Eurographics Association, Switzerland (2003) 89-96

8. Filip, J., Haindl, M.: Efficient image based bidirectional texture function model. In Chantler, M., Drbohlav, O., eds.: Texture 2005: Proceedings of 4th Internatinal Workshop on Texture Analysis and Synthesis, Edinburgh, Heriot-Watt University (2005) 7-12

9. Efros, A.A., Freeman, W.T.: Image quilting for texture synthesis and transfer. In Fiume, E., ed.: ACM SIGGRAPH 2001, ACM Press. (2001) 341-346

10. Cohen, M., Shade, J., Hiller, S., Deussen, O.: Wang tiles for image and texture generation. In: ACM SIGGRAPH 2003, ACM Press. Volume 22., New York, NY, USA (2003) 287-294

11. Kwatra, V., Schödl, A., Essa, I., Bobick, A.: Graphcut textures: image and video synthesis using graph cuts. ACM SIGGRAPH 2003, ACM Press 22(2) (2003) 277-286

12. Somol, P., Haindl, M.: Novel path search algorithm for image stitching and advanced texture tiling. In: Proceedings of 13-th International Conference in Central Europe on Computer Graphics, Visualization and Computer Vision, WSCG05. (2005)

13. Dong, J., Chantler, M.: Comparison of five 3D surface texture synthesis methods. In: Proceedings of the 3rd International Workshop on texture analysis and synthesis (Texture 2003). (2003) 47-52

14. Haindl, M., Filip, J.: A fast probabilistic bidirectional texture function model. In: Proceedings of International Conference on Image Analysis and Recognition. (Lecture Notes in Computer Science. 3212). Volume 2., Berlin Heidenberg, SpringerVerlag (2004) 298-305

15. Haindl, M., Filip, J., Arnold, M.: BTF image space utmost compression and modelling method. In: Proceedings of 17th International Conference on Pattern Recognition. Volume 3., IEEE Computer Society Press (2004) 194-198

16. Database, B.U.B. -URL: http://btf.cs.uni-bonn.de/- (2003)

17. Woodham, R.: Analysing images of curved surface. Artificial Intelligence 17(5) (1981) 117-140

18. Haindl, M.: Texture synthesis. CWI Quarterly 4(4) (1991) 305-331

19. Haindl, M., Šimberová, S.: A Multispectral Image Line Reconstruction Method. In: Theory \& Applications of Image Analysis. World Scientific Publishing Co., Singapore (1992) 306-315

20. Coxeter, H.S.M.: Introduction to Geometry. New York: Wiley (1969) 